\title{
Genetic Algorithm and Deep Learning feature based Tumor Detection
}

\author{
T. Balamurugan ${ }^{1}$, \\ Assistant professor, Department of ECE, Government college of Engineering, \\ Dharmapuri, Tamilnadu, India \\ balamurugan.thiyagu@gcedpi.edu.in \\ Dr.E.Gnanamanoharan ${ }^{2}$, \\ Assistant professor, Department of ECE, Annamalai University, \\ Chidambaram, Tamilnadu, India \\ gnanamanohar@gmail.com
}

\begin{abstract}
:
In medical diagnostics, brain tumour segmentation is a difficult process. The basic goal of brain tumour segmentation is to provide accurate characterizations of brain tumour regions by employing masks that are appropriately positioned. Deep learning approaches have shown a lot of promise in recent years for tackling issues like object recognition, picture classification, and semantic segmentation in computer vision. To obtain outstanding system performance in brain tumour segmentation, a variety of deep learning-based methods have been used. This article seeks to examine the recently created deep learning-based brain tumour segmentation technology in light of the most sophisticated technology and its performance. This work utilised a genetic algorithm based on fuzzy C-means (FCM-GA) to separate tumour areas from brain scans. During the preprocessing stage, the input picture is scaled to 256x256 pixels. A preprocessed MRI picture was segmented using FCM-GA. This is a sophisticated machine learning (ML) approach for finding items in huge datasets that is extremely flexible. To enhance the feature subset, the segmented picture is next subjected to hybrid feature extraction (HFE).Kernel Nearest Neighbor with a Genetic Algorithm (KNN-GA) is utilized in the feature selection process to achieve the optimum feature value. The RESNET classifier uses the best feature value to split the MRI picture into three regions: meningioma, glioma, and pituitary gland. The suggested hybrid method's performance is validated using real-time data sets. In comparison to current Convolutional Neural Networks (CNN) and Support Vector Machines (SVM) classification methods, the suggested technique enhances average classification accuracy by 7.99 percent
\end{abstract}

Keywords: Deep learning, Convolutional Neural Network, Genetic algorithm, Support vector machine, ResNet classifier,

\section{INTRODUCTION}

The segmentation of brain tumors is a significant priority in medical image processing (MIP). Early detection of brain tumors is crucial for improving options for treatment and maximizing patient survival rates. A large number of MRI images generated in clinical routines are manually segmented to diagnose cancer, which is a complex and time-consuming process. As a consequence, automatic brain tumor image segmentation is required.Computer-assisted intervention information services have long been regarded as an essential tool for medical imaging applications. Computer-aided assessment [1], health record data acquisition [2, 3], robotic surgery [4, 5] are all typical applications in necessary health diagnostics and therapy.

Medical image analysis would help healthcare professionals better understand their illness, investigate clinical problems, and enhance the quality of care. One of the primary motivations for this invention is that brain tumors can appear anywhere in the human brain and come in several different forms.

The low-quality imaging and diffusion boundary between abnormal and normal tissue also makes obtaining sufficient classification precision difficult. Many algorithms have been implemented for brain tumor segmentation to automatically extract feature and achieve statistical performance using powerful deep learning methods. Many issues remain unresolved in brain tumor segmentation. The goal of brain tissue segmentation also referred to as anatomical, aims to classify each voxel or pixel as an independent brain tissue category. According to this segmentation hypothesis, images of the brain do not contain problems such as tumor tissue $[7,8]$. There are no sub regions of the white matter lesion to divide using binary classification, such as necrosis and core. Tumor discovery seeks to detect an abnormal tumor tissue category. 
The detection result will be the bounding box, and the classification result will be the label [9-11]. It is worth noting that the term "brain tumour detection" used in some research work only returns the tumor tissue's bounding box as the detection result. Various studies on brain tumor detection have been conducted using genetic algorithms $[12,13]$ and convolutional neural networks $[14,15]$. Several research approaches return the single-label segmentation mask or the tumor core center as points of interest without further inference or segmentation.

\section{RELATED WORK}

In the healthcare field, experts have investigated several segmentation methods for detecting brain tumors. This chapter is a summary of several essential segmentation techniques. V. Jumb and colleagues, by combining Kmeans clustering and Otsu's adaptive threshold technology, propose colour image segmentation. The red-green-blue (RGB) image is converted to the HSV colour model in this work, and the channel value is extracted. To obtain a better threshold value from the image, we apply Otsu's multi-threshold value to the $\mathrm{V}$ channel. A morphological process is used for background subtraction in the final stage [16].

Among those who have contributed to this work are MdSujan et al. To detect brain tumors, we propose a new method based on morphological image analysis thresholds. The colour image is first converted to gray scale, and the noise in the gray scale image is then removed using filtering technology. To accurately segment the tumor area, we convert the grey image to a binary image and add a threshold of 0.3 to the Otsu threshold. Then, using morphological operations to locate the brain tumor visible in the brightest part of the MRI. Because it does not use any classification method, the proposed method's accuracy ( 84.72 percent) is ineffective in detecting brain tumors [17].

V. Anitha and others propose an adaptive pillar K-means (APKM) scheme. The classification method is divided into two levels. The two-layer classification method outperforms the SVM classifier method in terms of accuracy. The self-organizing map neural network (SOMNN) is trained using features extracted from the discrete wavelet transform hybrid wavelet (DWTBW), and the filter coefficients obtained from the KNN training are used in the proposed method. The testing procedure is divided into two steps. This method does not employ hybrid feature extraction technology to extract the tumor's affected area [18].

$\mathrm{N}$. Abiwinand and colleagues propose using $\mathrm{CNN}$ to classify brain tumors. $\mathrm{CNN}$ was used to categorize brain tumors into three types: glioma, meningioma, and pituitary gland. They used the most straightforward CNN architecture possible (for example, a complete connection comes from a convolutional layer, a top pooling layer, a flattening layer, and a hidden layer following it). CNN was trained on $3064 \mathrm{~T} 1$-weighted CEMRI images from a brain tumor dataset. In general, accuracy performance is critical for detecting brain tumors. However, the proposed method's accuracy has not been evaluated [19].

J. Chen et al. proposed a method to improve tumor classification performance. The image-enhanced expanded tumor area was initially used as a region of interest (ROI) rather than the original tumor area. Because the tumor surrounding the tissue can also provide important information about the type of tumor. The enlarged tumor area is divided into several small circular subsections in the second stage. However, the SVM classifier did not achieve highefficiency accuracy [20]. The proposed method is used to overcome the limitations above and improve the image segmentation process's performance.

\section{PROPOSED HYBRID DEEP LEARNING WITH GENETIC ALGORITHM FOR TUMOUR DETECTION}

Deep neural networks can thoroughly learn hierarchical features from input images rather than using predefined manual features. A concept comparison of a traditional brain tumor segmentation algorithm and a deep learning-based algorithm is shown in Figure 1.This algorithm necessitate a huge number of training data to solve the problem of over-compliance and a large amount of computing power to accelerate the training phase. When deep learning techniques are effectively integrated with weight initialization and optimization strategies, they achieve the best results.

This study uses the T1-W CEMRI data set to calculate the efficiency of the FCMGA-HFE-RESNET method. The size of the input image will be adjusted to $512 \times 512$. The preprocessed image is used in the segmentation process. Perform FCMGA to segment images with and without tumor cell areas. The FCMGA analyses local context 
information using the grey heterogeneity of adjacent images and wants to replace the standard Euclidean distance with a Gaussian radial basis kernel function. The flow diagram of proposed work is shown in figure. 2

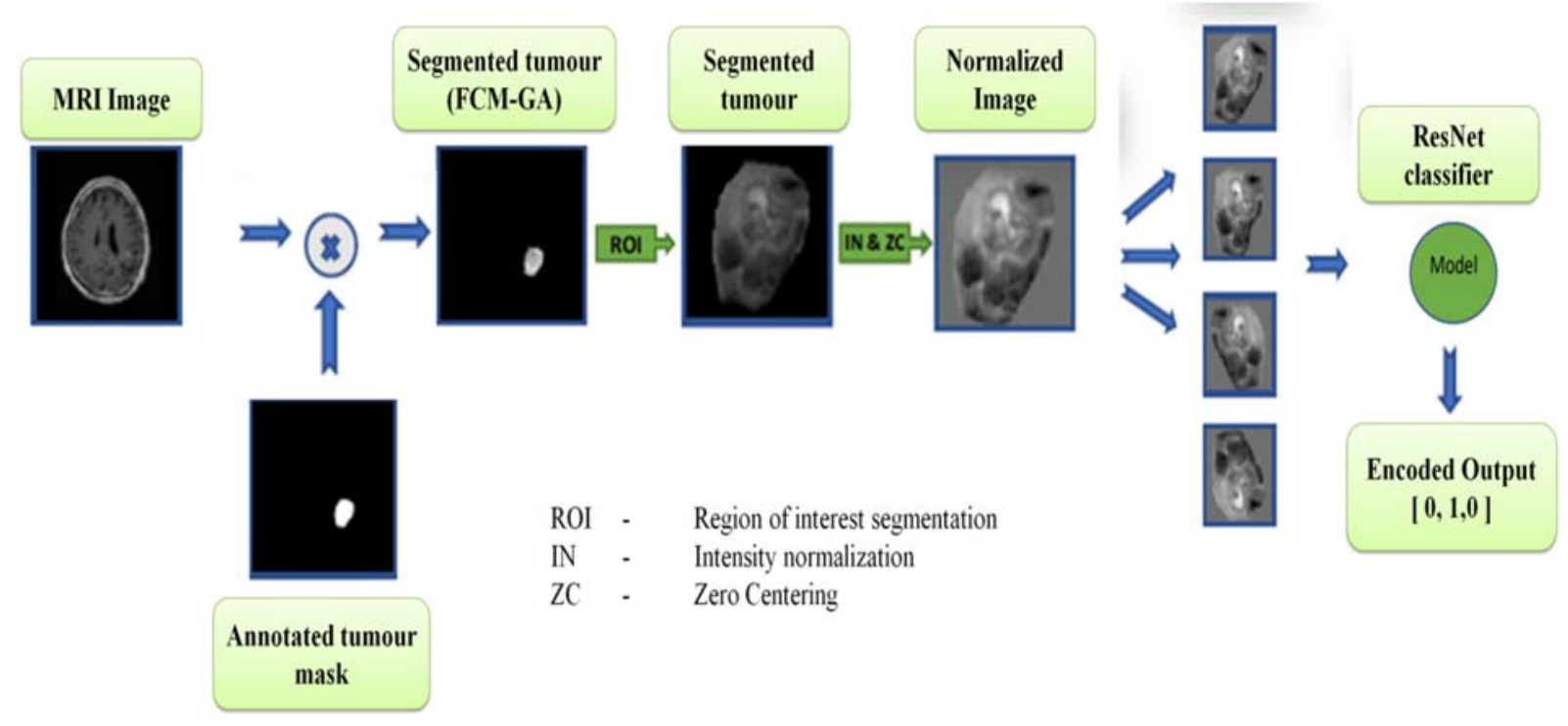

Figure 1. Overall Architecture of Brain tumor detection using hybrid approach

\subsection{Pre-Processing and Segmentation Process}

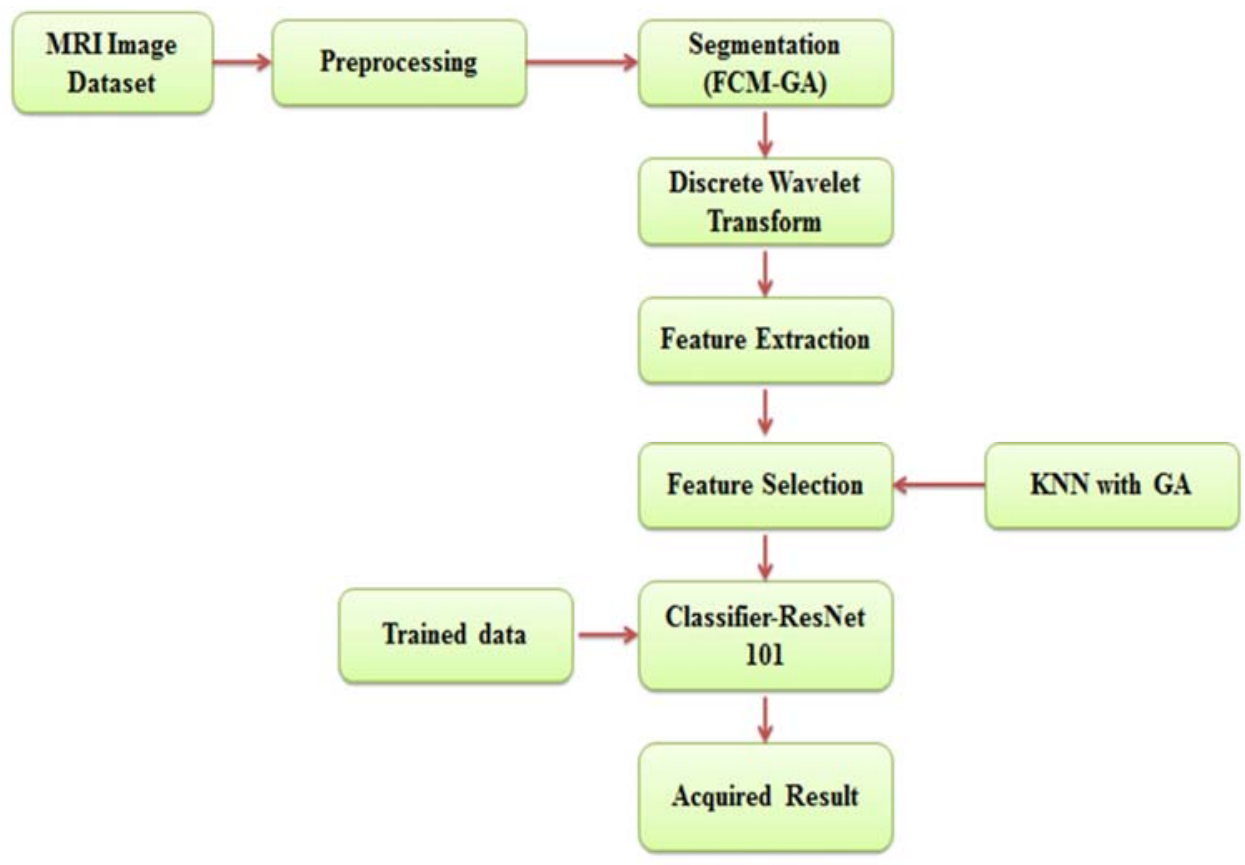

Figure 2. Proposed Block Diagram 


\subsection{Segmented image wavelet coefficient analysis using DWT}

The wavelet coefficients of the segmented image are calculated using DWT, which takes into account the square function. DWT technology has improved compression energy and reconstruction, no short support filter, a low calculation amount, and redundancy. DWT uses the fuzzy denoising procedure. This allows for movable sub-bands and improved direction selectivity while reducing redundancy. The actual texture of the image is calculated during the multi-resolution process by zooming in and out. The image is usually decomposed into several sub-images of varying resolution levels to store low-frequency and high-frequency information. A downsampler and a digital filter bank are used to realize 2D DWT. A digital filter bank that consists of a low-pass and a high-pass filter. The library numbers are organized by the wavelet configuration structure's required functions. The rows and columns of the brain tumor image are then processed separately using a one-dimensional wavelet transform to produce two-dimensional wavelet coefficients.

\subsection{Feature extraction}

This method extracts mixed features from the transformed brain tumor image. The action of mapping an image from image space to feature space is known as feature extraction. The histogram contains data about the edges and extensions of image discontinuities. LBP is the most widely used because it is simple to calculate features and is used in a wide range of facial recognition applications. Furthermore, the Histogram of Oriented Gradients (HOG) descriptor is used in image segmentation, and the computer version is used to extract the best feature value. These feature values are fed into the feature selection block as input for choosing the best feature.

\subsection{Feature selection using KNN based GA}

The feature selection method selects the optimum feature from the mixed feature extraction. Classification involves reviewing the dataset before sending the data to the classifier. Therefore, it helps to select essential and relevant functions in the detection of brain tumors. In addition, feature selection is used to find essential features in the classification process, reducing the classifier workload and improving classification accuracy.

In this study, KNN-based GA minimizes redundancy within input voxels and defines more correlations between input/output voxels in this study. The fitness function of the input voxel subset is then calculated using KNN, which maximizes the mutual data between voxels. Finally, to detect the most active voxels, we used a crossover operator and a mutation operator to decrease the overlap-based fitness function (FF). Some genetic algorithm features are preferred as chromosomes, and each chromosome is transferred to the $\mathrm{KNN}$, with the fitness value calculated.

KNN captures features by using each chromosome as a mask. KNN assigns fitness values to each chromosome and uses these values in the genetic algorithm chromosome calculation process. The genetic algorithm then finds the best subset of features in the final step.

\subsection{Segmentation using FCM-GA}

The genetic algorithm determines the best criterion value by simulating population evolution until the best individual survives. Individuals obtained from previous generations through crossover, mutation, and individual selections are known as survivors. GA believes that determining the best combination of segmentation results is a viable option for two reasons. The first reason is that it is difficult to distinguish between standards. GA is soft computing algorithm that does not require the fitness function to be distinguished; it simply evaluates it. Second, if the size of the exploration space is specified and the population is large enough, it is almost certain that the best fitness value will be obtained. GA is a type of local search. Essentially, a large number of synchronous agents (populations), each with an encoding state (chromosome), walk in the search space at random, combining existing solutions to form a new solution (crossover). Once discovered in a perfect area, the search work can be adjusted and refocused.

Several important decisions have been made to apply genetic algorithms based on encoding the population, mutating the population, and selecting parents. Crossovers (roulette, tournament selection), crossovers, and finally, the fitness function for performance evaluation. Although these options appear complex when the energy function has hundreds or thousands of dependent variables and parameters, some of them are nearly optimal for all relevant variables and parameters. Four steps are carried out to execution of GA. 
- Calculation of each individual's initial population (division result) and fitness function

- The result of the K-means division of image $\mathrm{S} 1$ is considered to be individually described by the class of each pixel.

- A collection of individuals characterized by genotype. It consists of split results to be combined.

- This feature can be used to quantify an individual's suitability for the environment, taking into account the individual's genotype.

There are two phases to the fitness computation process. Cluster formation is the first phase.This is done by assigning each point $x i, i=1,2 \ldots . n$ to one of the clusters $C j$ withcentrezjsuch that

$$
X_{i}-z_{J}<\left|X_{i}-Z_{p}\right|, p=1,2,3, \ldots . K, \quad p \neq j(1)
$$

After clustering, the cluster centres encoded in the chromosome are replaced by the mean points of the respective clusters. Cluster $\mathrm{Ci}$, the new centreZi* is computed as

$$
\begin{gathered}
Z_{i}^{*}=\sum_{x} C X_{i}, \quad i=1,2,3, \ldots . K \\
M=\sum_{i=1} M_{i}, \quad i=1,2,3, \ldots . K \\
M=\sum_{x_{i} C_{i}} \quad\left|X_{j}-Z-i\right|(4)
\end{gathered}
$$

\subsection{ResNet-101 classifier}

One of the most in-depth architectures proposed for ImageNet is the ResNet design (Object Detection and Image Classification Challenge). CNN typically trains multiple layers to communicate with one another and perform various tasks. The network is trained in several levels of functions at the end of the layer. The convolutional layer size of this approach includes three primary filters. ResNet layers have almost the same number of filters for the same output feature map size, and having the feature map size doubles the number of filters to keep all layers' time complexity constant.

The ResNet-101 model was built from the ground up and then fine-tuned to fit the training data. There is no data expansion at first, resulting in a total of 3064 samples, and then data expansion is used to increase the data size to 6744. The resnet is used in the proposed brain tumor classification method. The original data set contains images with a resolution of $512 \times 512 \times 1$, which are preprocessed using the method described above to produce an image with a resolution of $256 \times 256 \times 1$, focusing solely on the segmented tumor or ROI [20]. If the verification loss does not improve, the learning rate will be reduced by 0.2 to 0.001 every third epoch.

These regularization techniques are used to keep the model from becoming overfit. The network is trained without data expansion for 12,185 iterations before being trained again for 12,789 iterations. The preprocessed data set has been divided into training and testing parts.

\section{RESULT ANALYSIS}

To assess the performance of three-stage brain tumors such as glioma, meningioma, and pituitary gland, uses the feature selection shown in Table 1. In this study, we tested three feature extraction techniques that are fundamentally similar. The hybrid feature distinguishes the paired relationship between two adjacent pixels and yields better results. The arc histogram considers the relationship between multiple pixels by using image blocks as local features. As a result, Bow histograms are more discriminative and informative, resulting in the best results. Table 1 depicts the feature selection method's performance evaluation of stage 3 brain tumors. The performance analysis of stage 3 brain tumors without feature selection is shown in Table 2.

After segmentation, KNN-GA technology is used in this study to extract texture and direction gradient features from the segmented image. The texture feature is calculated using the statistical distribution of the intensity combination observed at each image's specified position. Based on a genetic algorithm, selects the best feature from the hybrid feature extraction. The classification considers the data set before sending it to the classifier.

Therefore, it helps to select more essential and relevant functions than existing methods in detecting brain tumors. After the feature selection process, the resnet classifier classifies tumor sites such as glioma, meningioma, and pituitary gland according to the selected features. Compared with the existing methods, the proposed method gives better results. In Table 1, the accuracy of the proposed method using feature selection is $93.21 \%, 99.54 \%$, and $99.87 \%$ 
in three different tumor classifications. Here, the proposed feature selection method is more effective in classifying meningioma and pituitary tumors than gliomas in terms of classification accuracy. In Table 2, without feature selection, the accuracy of the proposed method is $84.24 \%, 86.65 \%$, and $85.32 \%$, respectively, for three different tumor classifications.

$$
\begin{gathered}
\text { Specificity }=\frac{t n}{t n+f p} \\
F-\text { Score }=\frac{2 * \text { precision } * \text { recall }}{\text { precision }+ \text { recall }} \\
\text { Accuracy }=\frac{t p+t n}{t p+f n+t n+f p}
\end{gathered}
$$

wheretp $=$ true positive, $\mathrm{fp}=$ false positive, $\mathrm{tn}=$ true negative, $\mathrm{fn}=$ false negative
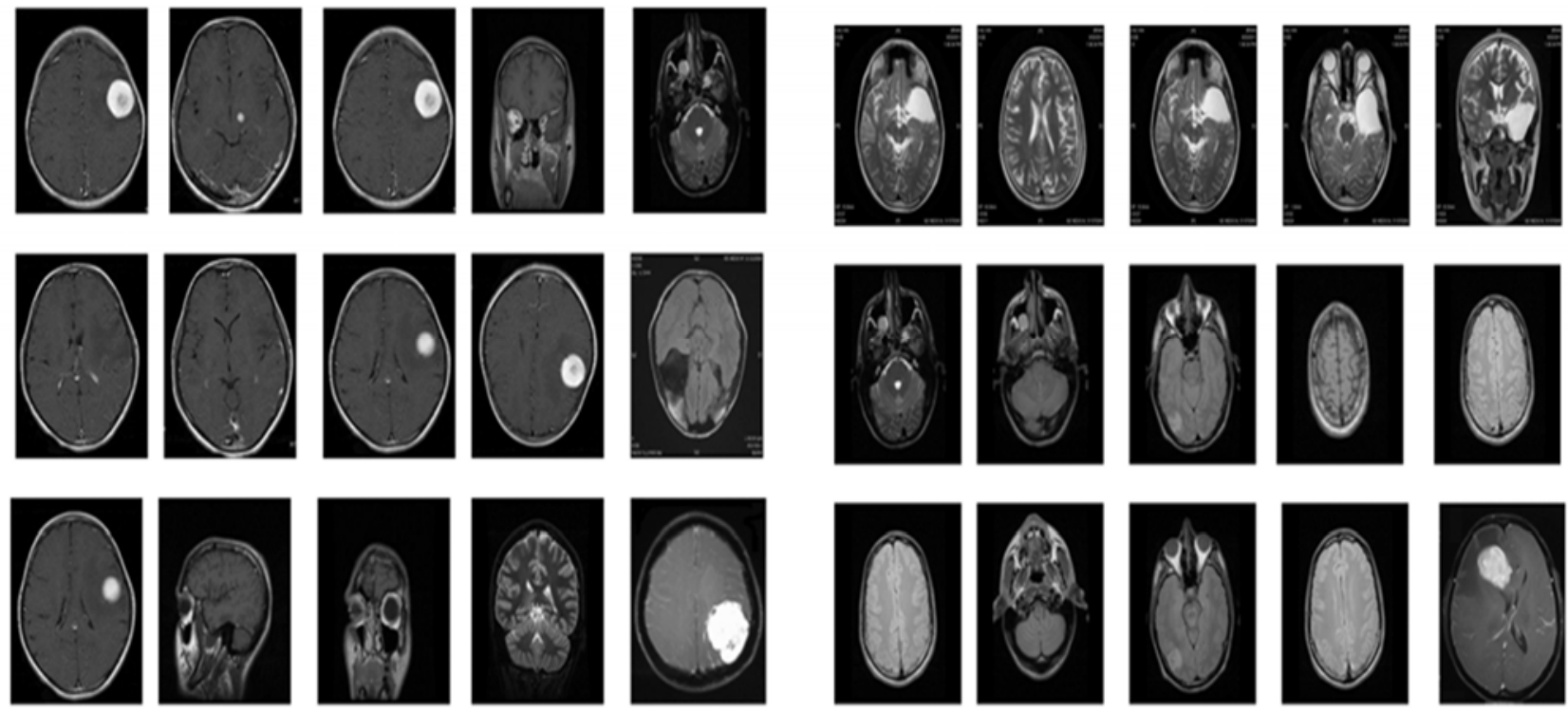

Figure 3. MRI images of Brain tumour dataset- I \& II 

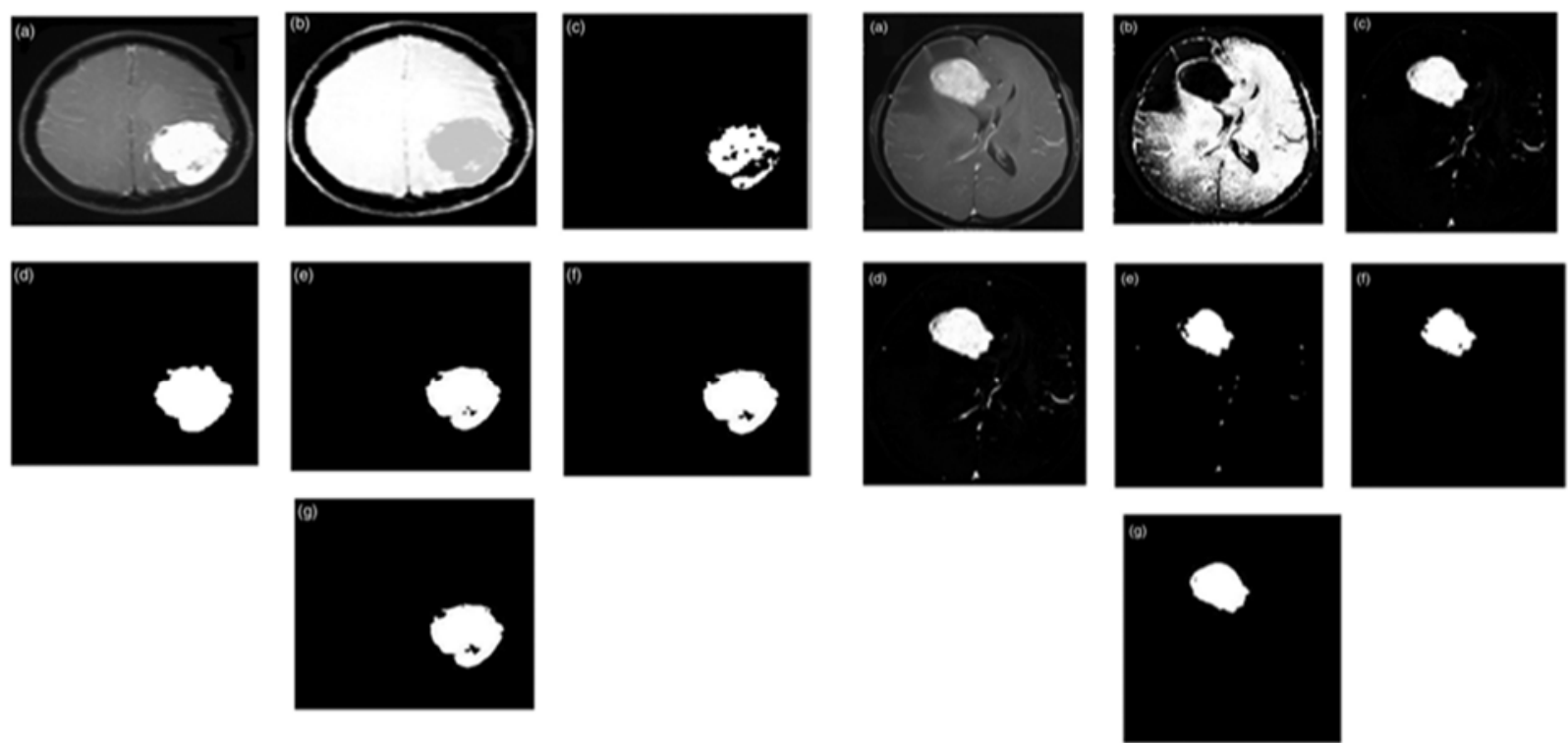

Figure 4. Segmented Output -Dataset I \& II

Table1: Performance evaluation of brain tumour classification of proposed approach

\begin{tabular}{|l|l|l|l|l|}
\hline \multirow{2}{*}{ Tumour Stage } & \multicolumn{3}{|c|}{ Performance Analysis with feature selection } \\
\cline { 2 - 5 } & Accuracy \% & Sensitivity \% & Specificity \% & Precision \% \\
\hline Glioma & $93.21 \%$ & $93.56 \%$ & $99.76 \%$ & $100 \%$ \\
\hline Meningioma & $99.54 \%$ & $99.96 \%$ & $99.98 \%$ & $100 \%$ \\
\hline Pituitary & $99.87 \%$ & $99.87 \%$ & $97.12 \%$ & $95.78 \%$ \\
\hline
\end{tabular}

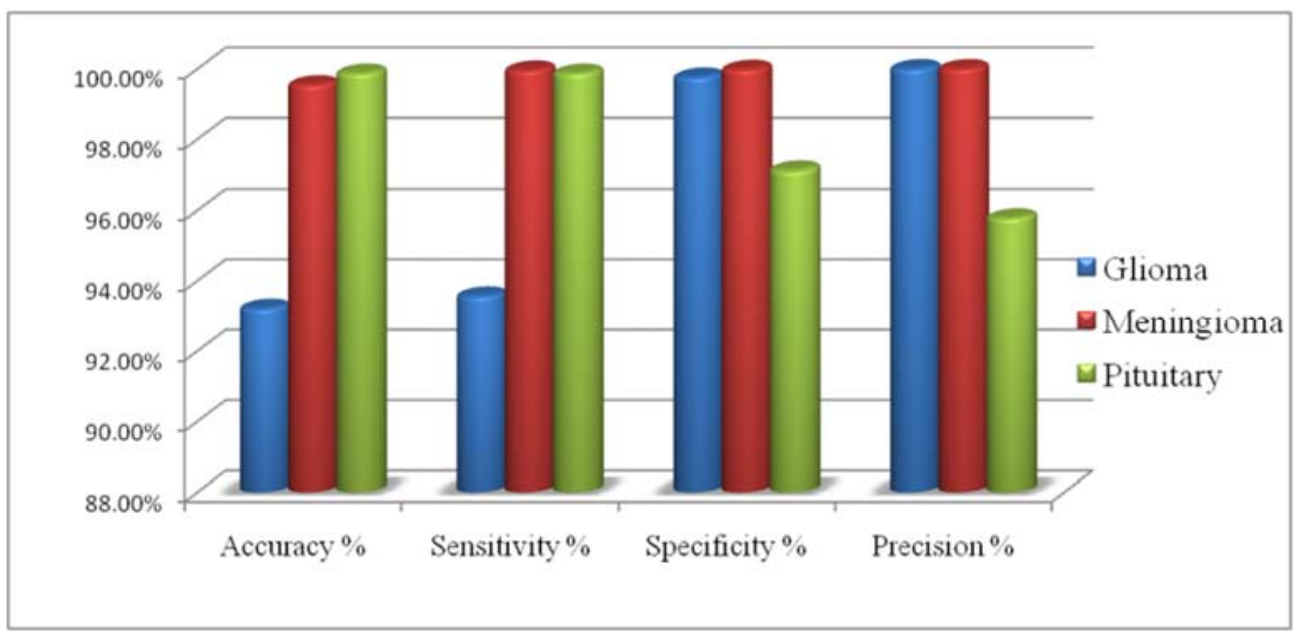

Figure5. Performance evaluation of brain tumour classification of proposed approach 
Table2: Performance evaluation of brain tumour classification without feature selection

\begin{tabular}{|l|llll|}
\hline \multirow{2}{*}{$\begin{array}{l}\text { Tumour } \\
\text { Stage }\end{array}$} & \multicolumn{4}{|c|}{ Performance Analysis without feature selection } \\
\cline { 2 - 5 } & Accuracy \% & Sensitivity \% & Specificity \% & Precision \% \\
\hline Glioma & $84.24 \%$ & $75.689 \%$ & $93.698 \%$ & $87.43 \%$ \\
\hline Meningioma & $86.65 \%$ & $79.56 \%$ & $88.28 \%$ & $75.14 \%$ \\
\hline
\end{tabular}

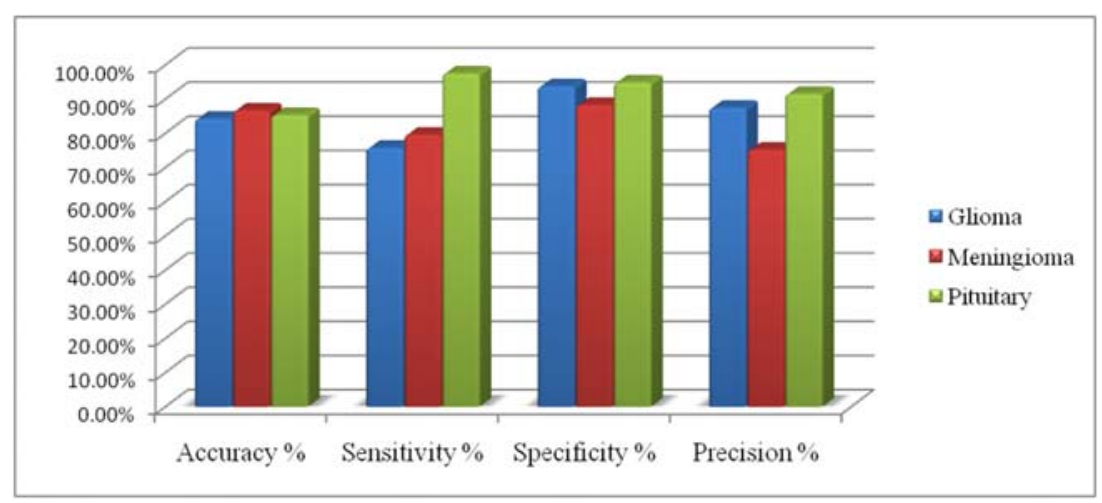

Figure 6. Performance evaluation of brain tumour classification without feature selection

Table.3 Performance evaluation of proposed approach with existing approach.

\begin{tabular}{|lllllll|}
\hline Algorithm & Recall & Precision & F-score & Accuracy & Sensitivity & Specificity \\
\hline SVM & 88.3 & 93.1 & 90.9 & 95.7 & 93.6 & 96.3 \\
\hline DECISION TREE & 91.8 & 94.8 & 92.3 & 93.2 & 95.4 & 95.7 \\
\hline CNN & 96.6 & 93.4 & 93.7 & 95.2 & 95.8 & 93.1 \\
\hline RANDOM FOREST & 98.4 & 96.6 & 94.5 & 96.8 & 97.2 & 98.1 \\
\hline GA & 95.8 & 92.7 & 91.8 & 92.5 & 91.3 & 92.6 \\
\hline FUZZY C MEANS & 94.1 & 91.3 & 92.4 & 91.1 & 92.6 & 93.4 \\
\hline PROPOSED METHOD & 100 & 97.9 & 98.5 & 99.3 & 98.5 & 97.9 \\
\hline
\end{tabular}

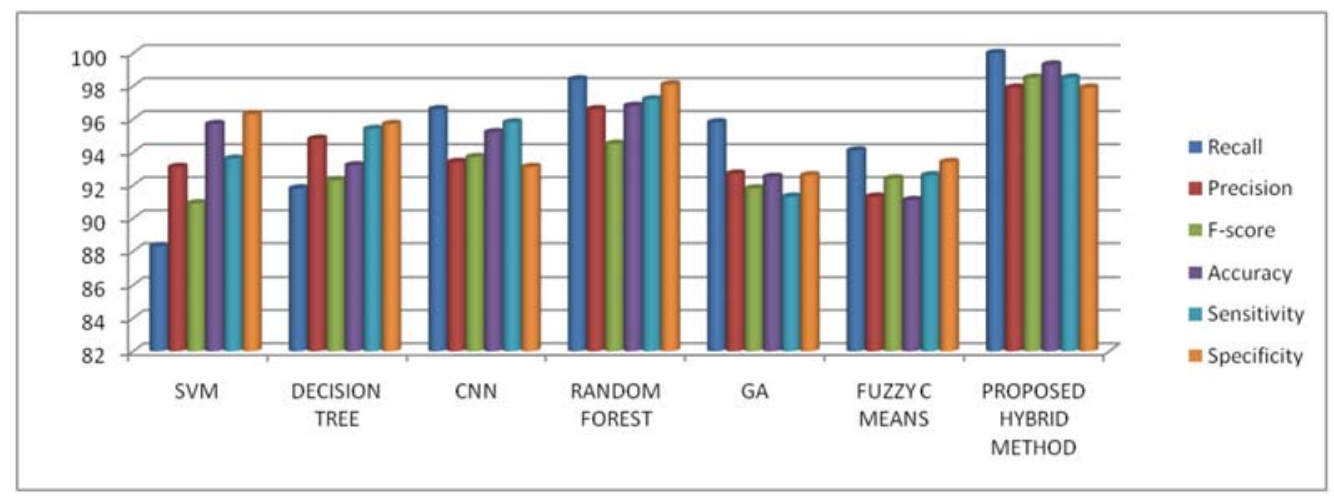

Figure 7. Comparison of performance evaluation

FCMGA can be used to segment MRI brain images. DNN is used to classify abnormal brain images into meningiomas, gliomas, and pituitary glands to determine classification accuracy. The experimental results show that, compared to existing methods, the proposed FCM-GA-RESNET method is more accurate for detecting and classifying abnormal brain tumors. The proposed method has $99.33 \%$ accuracy, which is higher than the accuracy of existing 
brain tumor detection methods. Figures 5-7 depict three different brain tumors' input, clustering, and segmentation images.

\section{CONCLUSION}

The image segmentation process is critical in medical image processing. FCMGA segmentation technology is used in this task to segment the brain tumor portion of the brain image. The optimal feature value is extracted from the segmented area by HFE. Following that, the feature selection method is applied to the feature extraction data to select the best features. Finally, use the RESNET classifier to classify the suspicious part based on the function. When compared to existing methods, the proposed method outperforms them with an accuracy of $7.99 \%$ t. The rate of accuracy is $99.33 \%$. It is very promising to classify abnormal brain tumors from MRI images of patients and classify various types of tumors using the proposed method via the medical diagnosis system. In the future, hybrid feature extraction using optimized algorithms could improve the detection of brain tumors in terms of accuracy, sensitivity, and specificity.

\section{REFERENCE}

[1] K. Doi, "Computer-aided diagnosis in medical imaging: historical review, current status and future potential," Computerized medical imaging and graphics, vol. 31, no. 4-5, pp. 198-211, 2007

[2] M. Lavin and M. Nathan, "System and method for managing patient medical records," Jun. 30 1998, uS Patent 5,772,585.

[3] R. H. Taylor, A. Menciassi, G. Fichtinger, P. Fiorini, and P. Dario, "Medical robotics and computer-integrated surgery," in Springer handbook of robotics. Springer, 2016, pp. 1657-1684.

[4] G. Litjens, T. Kooi, B. E. Bejnordi, A. A. A. Setio, F. Ciompi, M. Ghafoorian, J. A. van der Laak, B. Van Ginneken, and C. I. S'anchez, "A survey on deep learning in medical image analysis," Medical image analysis, vol. 42, pp. 60-88, 2017.

[5] D. N. Louis, A. Perry, G. Reifenberger, A. Von Deimling, D. Figarella- Branger, W. K. Cavenee, H. Ohgaki, O. D. Wiestler, P. Kleihues, and D. W. Ellison, "The 2016 world health organization classification of tumors of the central nervous system: a summary," Actaneuropathologica, vol. 131 , no. 6 , pp. $803-820,2016$

[6] W. Liu, D. Anguelov, D. Erhan, C. Szegedy, S. Reed, C.-Y. Fu, and A. C. Berg, "Ssd: Single shot multibox detector," in European conference on computer vision. Springer, 2016, pp. 21-37.

[7] B. Patenaude, S. M. Smith, D. N. Kennedy, and M. Jenkinson, "A bayesian model of shape and appearance for subcortical brain segmentation," Neuroimage, vol. 56, no. 3, pp. 907-922, 2011.

[8] A. de Brebisson and G. Montana, "Deep neural networks for anatomical brain segmentation," in Proceedings of the IEEE Conference on Computer Vision and Pattern Recognition Workshops, 2015, pp. 20-28.

[9] M. Ghafoorian, N. Karssemeijer, T. Heskes, M. Bergkamp, J. Wissink, J. Obels, K. Keizer, F.-E. de Leeuw, B. van Ginneken, E. Marchiori et al., "Deep multi-scale location-aware 3d convolutional neural networks for automated detection of lacunes of presumed vascular origin," NeuroImage: Clinical, vol. 14, pp. 391-399, 2017.

[10] Q. Dou, H. Chen, L. Yu, L. Zhao, J. Qin, D. Wang, V. C. Mok, L. Shi, and P.-A. Heng, "Automatic detection of cerebral microbleeds from mri images via 3d convolutional neural networks," IEEE transactions on medical imaging, vol. 35, no. 5, pp. 1182-1195, 2016.

[11] Q. Dou, H. Chen, L. Yu, L. Shi, D. Wang, V. C. Mok, and P. A. Heng, "Automatic cerebral microbleeds detection from MRI images via independent subspace analysis based hierarchical features," in Engineering in Medicine and Biology Society (EMBC), $201537^{\text {th }}$ Annual International Conference of the IEEE. IEEE, 2015, pp. 7933-7936.

[12] Kumar, Vemula\& Krishna, Kodali\&Kusumavathi, Sarukonda. (2019). Genetic Algorithm Based Feature Selection Brain Tumour Segmentation and Classification. International Journal of Intelligent Engineering and Systems. 12. 214-223. 10.22266/ijies2019.1031.21.

[13] Chandra, G. \& Rao, Kolasani. (2016). Tumor Detection In Brain Using Genetic Algorithm. Procedia Computer Science. $79.449-457$. 10.1016/j.procs.2016.03.058.

[14] S. Das, O. F. M. R. R. Aranya and N. N. Labiba, "Brain Tumor Classification Using Convolutional Neural Network," 2019 1st International Conference on Advances in Science, Engineering and Robotics Technology (ICASERT), 2019, pp. 1-5

[15] Wu, Wentao\& Li, Daning\& Du, Jiaoyang\& Gao, Xiangyu\&Gu, Wen \& Zhao, Fanfan \& Feng, Xiaojie\& Yan, Hong. (2020). An Intelligent Diagnosis Method of Brain MRI Tumor Segmentation Using Deep Convolutional Neural Network and SVM Algorithm. Computational and Mathematical Methods in Medicine. 2020. 1-10. 10.1155/2020/6789306.

[16] V. Jumb, M. Sohani, and A. Shrivas, "Color image segmentation using K-means clustering and Otsu's adaptive thresholding”, International Journal of Innovative Technology and Exploring Engineering, Vol.3, No.9, pp.72-76, 2014.

[17] MdSujan, N. Alam, S.A. Noman, and M.J. Islam, "A Segmentation based Automated System for Brain Tumour Detection", International Journal of Computer Applications, Vol.153, No.10, pp.41-49, 2016.

[18] V. Anitha and S. Murugavalli, "Brain tumour classification using two-tier classifier with adaptive segmentation technique", IET Computer Vision, Vol.10, No.1, pp.9-17, 2016.

[19] N. Abiwinanda, M. Hanif, S.T. Hesaputra, A. Handayani, and T. R. Mengko, "Brain Tumour Classification Using Convolutional Neural Network", In: Proc. of World Congress on Medical Physics and Biomedical Engineering, pp. 183-189, 2019.

[20] J. Cheng, W. Huang, S. Cao, R. Yang, W. Yang, Z. Yun, Z. Wang, and Q. Feng, "Correction: Enhanced Performance of Brain Tumour Classification via Tumour Region Augmentation and Partition”, PloS one, Vol.10, No.12, pp.e0144479, 2015. 


\section{Author's Profile}

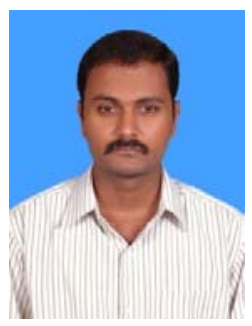

T.BALAMURUGAN received his Bachelor Degree in Electronics and Communication Engineering from Annamalai University, Chidambaram, Tamilnadu, India in 2006, and Master's Degree in Process Control and Instrumentation Engineering from Annamalai University, Chidambaram, and Tamilnadu, India in 2012. He is currently working as Assistant Professor in the Department of Electronics and Communication Engineering, Government college of Engineering, Dharmapuri, Tamilnadu, India. His current research areas are Digital image Processing, Sparse Signal Processing, Digital Communication and Wireless Communication.

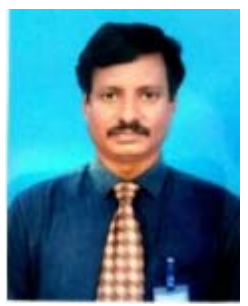

Dr. E.GNANAMANOHARAN, received the B.E degree in Electronics and Communication Engineering from Madras University, Chennai, Tamilnadu, India and M.E degree in Process Control and Instrumentation Engineering and Ph.D degree in Electronics and Communication Engg from Annamalai University, Annamalainagar, India. He is currently working as Assistant Professor of ECE at Annamalai University, Annamalainagar, India. His areas of interest include communication networks, wireless mobile ad hoc networks and wireless sensor networks. He has published 13 papers in various international journals. 